\title{
83 SPATIALLY RESOLVED TRANSCRIPTOMIC AND PROTEOMIC INVESTIGATION OF BREAST CANCER AND ITS IMMUNE MICROENVIRONMENT
}

Jennifer Chew*, ${ }^{1}$ Cedric Uytingco, ${ }^{1}$ Rapolas Spalinskas, ${ }^{1}$ Yifeng Yin, ${ }^{1}$ Joe Shuga, ${ }^{1}$ Benton Veire, ${ }^{1}$ Naishitha Anaparthy, ${ }^{1}$ Ryo Hatori, ${ }^{1}$ Anna-Maria Katsor, ${ }^{1}$ Layla Katiraee, ${ }^{1}$ Alexander Hermes, ${ }^{1}$ Jun Ding Chiang, ${ }^{1}$ Patrick Roelli, ${ }^{1}$ Stephen Williams, ${ }^{1}$ William Nitsch, ${ }^{1}$ Neil Weisenfeld, 'Dan Walkser, 'Jason Koth, ${ }^{2}$ Subham Basu, ${ }^{2}$ Will Howat, ${ }^{1}$ Karthik Ganapathy, ${ }^{1}$ Marlon Stoeckius. ${ }^{1} 10 X$ Genomics, Pleasanton, CA, USA; ${ }^{2}$ Abcam, Cambridge, UK

Background The tumor microenvironment (TME) is composed of highly heterogeneous extracellular structures and cell types such as endothelial cells, immune cells, and fibroblasts that dynamically influence and communicate with each other. The constant interaction between a tumor and its microenvironment plays a critical role in cancer development and progression and can significantly affect a tumor's response to therapy and capacity for multi-drug resistance. High resolution analyses of gene and protein expression with spatial context can provide deeper insights into the interactions between tumor cells and surrounding cells within the TME, where a better understanding of the underlying biology can improve treatment efficacy and patient outcomes. Here, we demonstrated the ability to perform streamlined multi-omic tumor analyses by utilizing the 10X Genomics Visium Spatial Gene Expression Solution for FFPE with multiplex protein enablement. This technique simultaneously assesses gene and protein expression to elucidate the immunological profile and microenvironment of different breast cancer samples in conjunction with standard pathological methods.

Methods Serial $(5 \mu \mathrm{m})$ sections of FFPE human breast cancer samples were placed on Visium Gene Expression (GEX) slides. The Visium GEX slides incorporate $\sim 5,000$ molecularly barcoded, spatially encoded capture spots onto which tissue sections are placed, stained, and imaged. Following incubation with a human whole transcriptome, probe-based RNA panel and an immuno-oncology oligo-tagged antibody panel, developed with Abcam conjugated antibodies, the tissues are permeabilized and the representative probes are captured. Paired GEX and protein libraries are generated for each section and then sequenced on an Illumina NovaSeq at a depth of $\sim 50,000$ reads per spot. Resulting reads from both libraries are aligned and overlaid with H\&E-stained tissue images, enabling analysis of both mRNA and protein expression. Additional analyses and data visualizations were performed on the Loupe Browser v4.1 desktop software.

Conclusions Spatial transcriptomics technology complements pathological examination by combining histological assessment with the throughput and deep biological insight of highly-multiplexed protein detection and RNA-seq. Taken together, our work demonstrated that Visium Spatial technology provides a spatially-resolved, multi-analyte view of the tumor microenvironment, where a greater understanding of cellular behavior in and around tumors can help drive discovery of new biomarkers and therapeutic targets.

http://dx.doi.org/10.1136/jitc-2021-SITC2021.083 